\title{
PIASA: A power and interference aware resource management strategy for heterogeneous workloads in cloud data centers
}

\author{
Altino M. Sampaio ${ }^{\mathrm{a}}$, Jorge G. Barbosa ${ }^{\mathrm{b}, *}$, Radu Prodan $^{\mathrm{c}}$ \\ a Instituto Politécnico do Porto, Escola Superior de Tecnologia e Gestão de Felgueiras, CIICESI, Felgueiras, Portugal \\ ${ }^{\mathrm{b}}$ LIACC, Departamento de Engenharia Informática, Faculdade de Engenharia, Universidade do Porto, Porto, Portugal \\ ${ }^{\mathrm{c}}$ University of Innsbruck, Institute of Computer Science, Innsbruck, Austria
}

\section{A R T I C L E I N F O}

\section{Article history:}

Received 16 February 2015

Received in revised form 30 June 2015

Accepted 1 July 2015

Available online 17 July 2015

\section{Keywords:}

Performance interference

Energy efficiency

CPU-intensive load

I/O intensive load

SLA

QoS

\begin{abstract}
A B S T R A C T
Cloud data centers have been progressively adopted in different scenarios, as reflected in the execution of heterogeneous applications with diverse workloads and diverse quality of service (QoS) requirements. Virtual machine (VM) technology eases resource management in physical servers and helps cloud providers achieve goals such as optimization of energy consumption. However, the performance of an application running inside a VM is not guaranteed due to the interference among co-hosted workloads sharing the same physical resources. Moreover, the different types of co-hosted applications with diverse QoS requirements as well as the dynamic behavior of the cloud makes efficient provisioning of resources even more difficult and a challenging problem in cloud data centers. In this paper, we address the problem of resource allocation within a data center that runs different types of application workloads, particularly CPU- and network-intensive applications. To address these challenges, we propose an interference- and power-aware management mechanism that combines a performance deviation estimator and a scheduling algorithm to guide the resource allocation in virtualized environments. We conduct simulations by injecting synthetic workloads whose characteristics follow the last version of the Google Cloud tracelogs. The results indicate that our performance-enforcing strategy is able to fulfill contracted SLAs of real-world environments while reducing energy costs by as much as $21 \%$.
\end{abstract}

(c) 2015 Elsevier B.V. All rights reserved.

\section{Introduction}

There is growing interest in the use of cloud computing, which has been progressively adopted in different scenarios, such as business applications, social networking, scientific computation and data analysis experiments [18,38]. However, clouds now host a wider range of applications with diverse resources and QoS requirements. From the consumers viewpoint, it is essential that the cloud provider offer guarantees about service delivery. Typically, consumers detail the required service level through QoS parameters, which are described in service level agreements (SLAs) established with providers [25]. More concretely, SLAs specify all the expectations and obligations of the service to be provided in terms of metrics as well the penalties for violating those expectations agreed upon by all parties. Thus, SLAs are a key element for supporting and empowering QoS in cloud environments.

\footnotetext{
* Corresponding author.

E-mail addresses: ams@estgf.ipp.pt (A.M. Sampaio), jbarbosa@fe.up.pt (J.G. Barbosa), radu@dps.uibk.ac.at (R. Prodan).
} 
Providing QoS guarantees in current cloud data centers is a very difficult and complex task due to the dynamic nature of the environment and the applications workload characteristics. The problem becomes even more complicated when considering efficient resource usage and technological limitations. Cloud computing environments are very dynamic by nature in that end customers share a large, centrally managed pool of storage and computing resources. At any one time, a substantial number of end users can be inactive (e.g., submitted jobs just finished, low utilization due to specific day of the week), which allows a service provider to opportunistically consolidate, multiplex and even transfer resources among virtual machines rented to different users. Moreover, as operational costs become more relevant, the implementation of policies for maximizing the efficiency, cost-effectiveness, and utilization of resources becomes paramount. However, balancing QoS guarantees with efficiency and utilization becomes extremely challenging because virtualization does not guarantee performance isolation between VMs. For example, an applications performance can change due to the existence of other co-resident VMs that share the last-level cache (LLC). This phenomenon is known as performance interference $[13,15,19,24]$. Furthermore, different applications demand different QoS requirements. For example, non-interactive batches require completion time, while transactional web applications are concerned with throughput guarantees. Different application workloads demand a diverse type and amount of resources. In particular, batch jobs tend to be relatively stable, while web applications tend to be highly unpredictable and bursty [10].

In this paper, we present a dynamic resource management strategy that optimizes power efficiency and considers the SLAs of two different types of workloads: CPU-bound (i.e., batch jobs) and network I/O-bound (i.e., transactional web) applications. We propose a mechanism that estimates the slowdown in co-hosted deadline-driven CPU-bound applications due to contention in on-chip resources and a second mechanism that responds to changes in demand for network I/O-bound applications. A scheduler algorithm is also proposed to compensate for deviations from the required performance in both types of applications. The algorithm applies readjustments in the VM to PM mapping to correct such performance deviations. The assignment of resources to VMs is also refined to optimize the energy efficiency of the underlying infrastructure.

The rest of the paper is organized as follows. Section 2 discusses related work in the area of resource provisioning in cloud data centers. Section 3 introduces the architecture of the power- and interference-aware mechanism to address the QoS of heterogeneous workloads. Section 4 considers the metrics used to assess the performance of the proposed mechanism and describes the workloads and performance deviation characteristics. Section 5 presents and discusses the results. Finally, Section 6 concludes the paper and discusses future research directions.

\section{Related work}

Energy efficiency optimization and assurance of application performance have two opposite objectives. While energy wastages can be mitigated through consolidation, performance deviations are caused by interference among co-hosted applications due to technological limitations (i.e., inefficient virtualization isolation) or by abrupt demand variation in the workloads. The occurrence of either makes the previous provisioning of resources inappropriate, and corrective actions must be performed to fulfill the application QoS requirements. This section presents notable efforts to understand the causes of performance deviation in co-hosted applications and to define strategies to guarantee QoS requirements for diverse applications while maximizing energy efficiency during runtime.

\subsection{Performance interference estimation in virtualized environments}

Determining the relationship between allocated resources and high-level metrics in a dynamic cloud environment is not trivial and has led to intensive research in related topics. For example, Koh et al. [19] have studied this phenomenon by measuring the effects of the consolidation of two VMs running diverse resource-bound applications (i.e., $\mathrm{CPU}$, memory, and $\mathrm{I} / \mathrm{O}$ ). Key findings include the following: (i) the performance of $\mathrm{I} / \mathrm{O}$-bound applications degrades much less when co-located with CPU- or memory-bound applications; (ii) CPU- and memory-bound applications consume a large amount of CPU resources; and (iii) the correlation between workload characteristics and performance is not linear. Huang and Lee [15] analyzed the adverse impact of performance interference from a security perspective. For this purpose, the authors exhaust one type of hardware resource by co-locating a misbehaved VM with the victim VM in different configurations. The results show the following: (i) the TCP throughput can fall below 70\% when the malicious VM uses memory intensively; (ii) the CPU execution time increases by $60 \%$ when a malicious VM uses the disk I/O intensively; and (iii) the memory bandwidth for the victim VM decreases from $20 \%$ to $80 \%$ when the malicious VM heavily uses disk I/O (the decrease is closer to $80 \%$ when the malicious VM uses network I/O). Mars et al. [24] explored the impact of contention in cache, memory bandwidth, and prefetcher resources in the performance of different types of applications. The authors showed that when the pressure in the cache exceeds saturation, the impact on an application QoS no longer increases. The experiments used real Google workloads and took place in a Google cluster. In addition, Hashimoto et al. [13] measured the performance degradation due to contention in network $\mathrm{I} / \mathrm{O}$, disk I/O, and on-chip resources. The results indicated that an application that uses more resources degrades more, and the impact on performance can change between $1 \%$ and nearly $190 \%$. Additionally, the results indicate that running two programs with high memory usage or network I/O causes high overhead in the hypervisor. Kousiouris et al. [20] studied the effect of critical parameters on the performance of VMs. Their study concluded that there is a nearly linear relationship between CPU share (i.e., quantum) and performance improvement. Additionally, application performance can 
degrade by as much as $160 \%$. The co-location of applications that are more CPU-intensive and less data-intensive results in the best performance, and the greatest impact on the performance of applications is from contention in LLC and memory bus resources. These studies have contributed important insights into the characteristics of applications and their relevance for co-hosting. This paper is complementary to these studies in that it uses their conclusions to better allocate resources to applications.

\subsection{Interference-aware dynamic resources scheduling}

With the objective of mitigating the performance degradation due to contention in hardware resources, several works have proposed quantifying and predicting the effects of interference from different types of resources so that appropriate actions can be taken by readjusting the map of VMs to physical resources. For example, Dwyer et al. [8] contributed a tool that measures the slowdown in the execution of CPU-bound applications. The slowdown is caused by the performance interference among co-located workloads that share LLC resources. The authors report that the tool is able to determine the degradation within $16 \%$ of the true value, on average. The approach does not consider workloads with different QoS requirements. Govindan et al. [11] proposed Cuanta, a practical technique for predicting performance degradation due to a shared processor cache for any possible placement of applications. The authors claim that the technique can be used to select the most efficient consolidation pattern according to required performance and resource constraints, achieving an average prediction error of less than 4\%. Unlike our work, the root cause of performance deviation is due to cache interference only. Chiang and Huang [7] developed the Tracon framework to mitigate the interference effects from concurrent data-intensive applications. Their aim was to reduce the runtime and improve the I/O throughput for data-intensive applications in a virtualized environment. Based on the application performance inferred from resource consumption observed from different VMs, the framework readjusts the provision of resources by invoking the interference-aware scheduler. Experiments considering data-intensive applications (e.g., bioinformatics, data mining, and video processing) showed that the framework is able to improve the application runtime by $50 \%$ and the $\mathrm{I} / \mathrm{O}$ throughput by as much as $80 \%$ for data-intensive applications executing in VMs. The framework is interference-aware biased and, hence, does not implement energy-efficient strategies. Lim et al. [22] proposed the D-factor model, which models the problem of performance interference within a physical server based on a collection of multiple resource queues for which contention creates non-linear dilation factors in jobs. The authors test CPU- and disk I/O-bound applications. Their key findings are that (i) multiple-resource contention creates non-linear dilation factors in jobs; (ii) there is a linear relationship between total completion time and individual completion times for the same type of job; and (iii) the co-location of workloads utilizing different system resources leads to the best efficiency. The authors claim that the proposed D-factor model is able to predict the completion time of coexisting workloads within a $16 \%$ error for realistic workloads. Our work is complementary in that it adds a mechanism to make decisions based on noisy performance deviation data samples to fulfill diverse contracted SLAs according to mixed/heterogeneous application types and requirements, and it tries to optimize energy efficiency at the same time. Nathuji et al. [29] proposed Q-Clouds, a QoS-aware control framework that transparently provides additional resources as necessary to achieve a performance similar to that if the applications were running in isolation. The framework dynamically provides underutilized resources to improve system efficiency (in terms of the runtime of CPU-bound applications). A common problem in these studies is that they either do not consider energy efficiency or their contribution to achieving it is unclear. Verma et al. [40] performed an experimental study about application performance isolation, virtualization overhead with multiple VMs, and scenarios in which applications are isolated from each other. From the insights obtained, the authors propose a framework and methodology for power-aware application placement for high-performance computing (HPC) applications that considers both CPU and cache size constraints. However, the work is based only on contention over CPU and LLC resources. The approach does not consider auto-scaling requirements of applications, nor does it readjust the provisioning of resources in the case of failure to meet the application QoS requirements. In [39], enterprise server web-based workloads were studied for the purpose of finding key characteristics for semi-static and static consolidation. The observations indicated that the correlation between workloads should be considered so that SLA violation can be avoided when such workloads are placed together. Based on these findings, the authors proposed two consolidation algorithms, namely, Correlation-Based Placement (CBP) and Peak Clustering-Based Placement (PCP). While the former adds co-location constraints between correlated applications to ensure that an application can be sized based on an off-peak value, the latter reserves additional capacity for each workload according to the size of the application to accommodate their maximum peaks. The study target was specific enterprise applications for which load behavior could be predicted for long periods, such as a day or a week, and statistical analysis was used to statically allocate applications to resources for long periods. In a dynamic system, such as the one considered in this paper, the statistical approach to determine capacity constraints is no longer valid [39], and therefore, we cannot directly compare our results to CBP and PCP algorithms. However, one of the main conclusions of the Verma et al. [39] study is that uncorrelated loads should be allocated in the same server. This feature has been followed in subsequent works, such as [10], and it is applied in our algorithm.

\subsection{SLA-based resources scheduling}

Other approaches for scheduling applications and provisioning resources in cloud computing environments consider the diversity of applications in terms of workload characteristics and QoS requirements. To this end, Rao et al. [31] developed a 
QoS provisioning framework that provides adaptive multi-objective resource allocation and the service differentiation of multiple applications. It applies a hard limit over the CPU assigned to an application. However, the work does not consider the effective utilization of shared virtualized resources, it is limited to the regulation of a single resource, and it only considers the response time and deviation from the Service Level Objective (SLO) value. While their work focuses on responding to abrupt workload or capacity changes, our study aims at increasing profit by diminishing energy consumption. It also addresses the problem of resource allocation within a data center that runs different types of application workloads that are subject to performance deviations due to interference among co-hosted applications. Moreover, we consider different types of QoS requirements, and we extend the strategy of the hard limit by applying hard and soft limits to address the two types of resources, CPU and I/O bandwidth, in the management of network-intensive workloads. Garg et al. [10] proposed an admission control and scheduling mechanism to maximize the resource utilization and profit. At the same time, it ensures that the QoS requirements of users are met as specified in the SLAs. The mechanism considers multiple types of SLAs based on application requirements (i.e., two types of applications, namely, non-interactive and transactional). Our work is closely related to [10] in that we address the resource allocation problem within a data center that runs different types of application workloads. Therefore, we compare our algorithm to this work.

\subsection{List of contributions}

This paper proposes a mechanism to efficiently detect and mitigate occurrences of performance deviation during application runtime, which cause non-observation of application QoS requirements, while optimizing the energy efficiency of the system. The main contribution of this work is the design and implementation of a mechanism with the following features:

- A scheduling strategy for heterogeneous workloads in virtualized data centers, with different characteristics and specific SLA requirements.

- A mechanism to detect deviations in application performance requirements, even when performance data samples contain noise.

- Consideration of multiple types of SLAs, according to mixed/heterogeneous application types and requirements.

- Support for auto-scaling of resources to satisfy SLAs and cope with peak time demands.

- Support for mitigation of performance degradation caused by contention due to sharing of physical resources among co-hosted applications.

- Optimization of energy efficiency by maximizing the utilization of cloud resources.

- In addition to the above mechanism features, this work contributes a study of the trade-off between the opposing objectives of energy efficiency and performance improvement.

\section{System model}

This section provides a formal description of a power- and interference-aware cloud architecture that dynamically maps VMs to PMs to improve both applications' QoS requirements and energy efficiency.

\subsection{Cloud data center overview}

We consider a private data center environment consisting of identical physical servers. It is assumed that each server is interconnected with a high-speed LAN network and high-bandwidth link to the Internet. The cloud provider manages its resources (e.g., virtual machine migration, signaling and control data) using a high-speed LAN link $N_{1}$, while the Internet link $N_{2}$ is utilized by cloud users to access cloud services. In this regard, overloading interface $N_{1}$ manifests itself in the overhead of VM migration, while interface $N_{2}$ is one of the resources VMs directly utilize so cloud users can access the deployed services. Each physical host in the cloud infrastructure has the same CPU capacity $C$, memory capacity $R$, LAN network bandwidth $N_{1}$, Internet network bandwidth $N_{2}$, access to a shared storage space $B$ for storing the disk images of the VMs, and an LLC $L$, such that for each physical server, $S_{i}=\left\{C, M, N_{1}, N_{2}, B, L\right\}$. In a typical usage scenario, users request services to deploy applications, and the cloud manager is responsible for evaluating the capacity required and for deciding whether to accept or reject the service requests according to the servers' ability to guarantee QoS requirements. The cloud architecture, information flow, and key components involved in performance estimation, power-efficiency, and the scheduling of applications are illustrated in Fig. 1 [34]. For simplicity, a VM encapsulates one single application or task and is the unit of migration in the system. Each application can be described by one of two possible types of workloads according to the most consumed resources: CPU-bound or network I/O-bound. Each type has different behaviors, characteristics, and QoS requirements. Each VM will run on top of one PM at a time, and multiple distinct VMs can be mapped to a single PM. The set of VMs constitutes the user's virtual cluster execution environment. The two modules, power efficiency and performance deviation, continually monitor servers and running applications and dynamically update the maps of VMs to PMs to maintain the energy efficiency of the system and to fulfill the application's QoS requirements. 


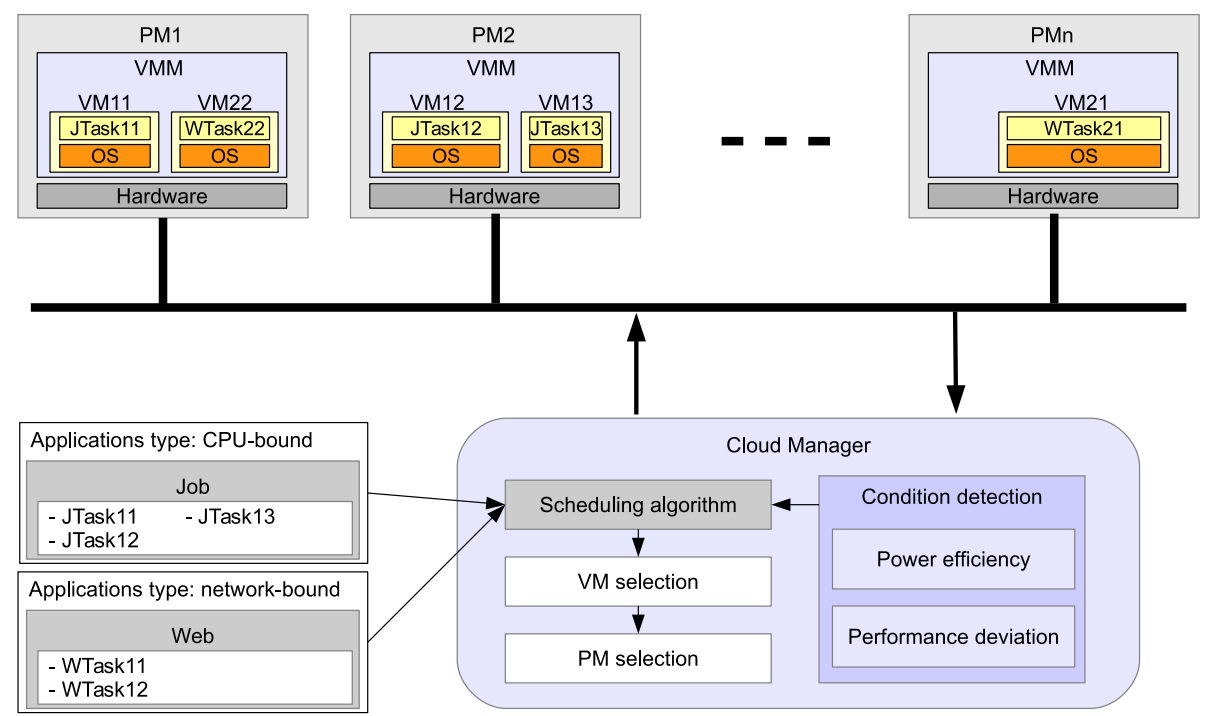

Fig. 1. Private cloud management architecture.

\subsection{Application overview}

In this study, we consider two types of workloads that can be deployed by users in the cloud infrastructure, namely, CPU-bound and network-bound workloads.

\subsubsection{CPU-bound workloads}

Scientific computing involves the construction of mathematical models and numerical solution techniques to solve scientific, social and engineering problems. Recently, scientific computing has started to explore clouds in alternatives to traditionally dedicated HPC infrastructures $[38,18]$. We model the execution of scientific applications as the problem of dynamically scheduling non-interactive batch jobs with specific CPU needs. Each job $j=\left(\Gamma_{j}, d_{j}\right)$ is composed of a set of $l$ independent CPU-intensive tasks, $\tau_{q} \in \Gamma_{j}, q \in\{1, \ldots, l\}$, and a deadline, $d_{j}$. Here, the deadline is used as the QoS requirement for batch jobs. The task workload is expressed in Mflops. Once tasks are independent, the job deadline becomes the deadline of the longest task. Job deadlines become activated as soon as cloud users submit jobs.

The submitted jobs and respective sets of tasks have heterogeneous resource requirements and varying deadlines. As documented by Stillwell et al. [37], a task $\tau$ can consume at most a maximum amount of resources $\max r(\tau)$ to execute at the maximum speed. The value of this parameter is defined by the user. In turn, the deadline $d_{\tau}$ to conclude the task defines the minimum execution speed and simultaneously constrains the minimum amount of resources $\min r(\tau)$ to assign, as defined by Eq. (1). The amount of resources assigned to a task is expressed in Mflops/s.

$$
\min r(\tau) \geqslant \frac{W(\tau, t)}{d_{\tau}-t-m_{e i}}
$$

where $t$ is the current time, $W(\tau, t)$ is the remaining workload for task $\tau$ at instant $t$, and $m_{e i}$ represents the time overhead required for a VM to migrate from node $e$ to node $i$. Parameter $m_{e i}$ equals 0 only when the task is scheduled for the first time.

The slack time of task $\tau$ is stated by Eq. (2) and defines the amount of extra time needed to complete the task by the ensemble deadline $d_{\tau}$.

$$
\text { slack_time }=\left(d_{\tau}-t-m_{e i}\right)-\frac{W(\tau, t)}{\max r(\tau)}
$$

Those tasks for which Eq. (2) returns a negative value are cancelled, and the corresponding jobs are considered incomplete, incurring an SLA violation. Assigning each task a CPU power between $\min r(\tau)$ and $\max r(\tau)$, we are fulfilling the deadline constraint given by Eq. (3) and, hence, satisfying the strict SLAs imposed on each job:

$$
F T(\tau) \leqslant d_{\tau}
$$

where $F T(\tau)$ is the finish time of task $\tau$. Due to interference caused by the sharing of on-chip resources (e.g., CPU, LLC, and memory bandwidth), co-hosted tasks can suffer from performance degradation, which induces a slowdown of running applications, ultimately resulting in a deviation between expected and delivered QoS. 


\subsubsection{Network-bound workloads}

Commonly commercial clouds have been built to support web and small database workloads [16]. Web systems are characterized by their dynamic changing of incoming workloads and VM resource variation and do not have a requested time or deadline. Mars et al. [24] propose that one way to measure the QoS of web servers is to measure the server throughput at different workload rates, namely, the maximum number of successful queries per second, as is the case in Google's web search. According to Mei et al. [26], the performance of web servers is CPU bound under a mix of small files and is network bound under a mix of large files. This work considers the second case by assuming that web workloads are mainly characterized by network resource consumption (i.e., are network-intensive) and have residual CPU consumption. The CPU time consumed to process network requests can be divided into two major categories: the time spent establishing TCP connections and the time spent transporting web file content [26]. Because the demand for resources changes abruptly with time, the amount of transferred network I/O $b(\tau)$ served by a task $\tau$ at each instant is given by Eq. (4).

$$
b(\tau)=\sum_{y=1}^{r e q / t u} h_{y}
$$

where $h_{y}$ is the file size to transfer in each request $y$. For each task, the number of requests per time unit req/tu varies with time, and the size of the file to transfer $h_{y}$ in each request is assumed to be large and practically constant. Due to the fluctuating nature of web-based transactional workloads, the cloud must adapt to application demands by scaling dynamically and providing resources accordingly. For instance, a general web application presents a varying workload according to the number of users accessing it. The SLA agreement defines the performance requirement of web applications in terms of requests served within a certain period of time. The performance requirement can be translated to CPU capacity $r(\tau)$ and network I/O bandwidth $b(\tau)$ [6]. We assume that a request is not served by the web application if the CPU $r(\tau)$ or network bandwidth $b(\tau)$ capacity allocated to task $\tau$ is less than the required capacity at instant $t$. The QoS requirements are violated if a certain number of non-served requests, from the total req/tu submitted to the web application within a time interval, exceeds the maximum specified by the SLA.

\subsection{Cloud manager}

The cloud manager is the main component in the cloud computing management infrastructure. Its function is to continually obtain the virtual and physical machine statuses, such as power consumption, and performance data to infer possible deviations in the task QoS requirements. Then, based on the information collected, the cloud manager makes decisions concerning opportunities to improve power efficiency or the need to readjust the allocation of resources to correct possible performance deviations. Energy efficiency is achieved by means of consolidation, transferring VMs from lower loaded PMs to other PMs so that the first PMs can transit to sleep mode. This method improves resource utilization by increasing the load rate of the active PMs [34], but it can result in high levels of performance degradation. In turn, performance deviations can be estimated based on techniques that differ according to the type of application under consideration.

\subsubsection{Power-efficiency logic}

An idle server represents $60-70 \%$ of the power consumed when it is fully utilized $[12,43]$. The power consumption in a computer node is mainly dictated by the CPU resource [3,4]. Thus, we consider only the CPU power consumption in our energy model to consolidate running VMs in servers. Then, a reduction in power consumption can be effectively achieved by switching idle PMs to sleep mode, incurring near-zero overhead [27]. The linear power model in Eq. (5) estimates the power consumption $P_{i}$ for a $P M_{i}$.

$$
P_{i}=p 1+p 2 \times C P U_{i}
$$

where $C P U_{i}$ is the CPU utilization for a given time interval, measured for a PM $i$ at runtime, and varies within $[0,1]$. The $p 1$ and $p 2$ factors are the power consumption when PM is idle and the additional power consumption due to CPU utilization, respectively. Eq. (6) is the power efficiency of a PM $i$ at a specific time interval and reflects how much useful work is produced for a given power consumption [42].

$$
E_{P_{i}}=\frac{C P U_{i}}{p 1+p 2 \times C P U_{i}} \times(p 1+p 2)
$$

increases monotonically with CPU utilization, reaching 1 when CPU is 1 . Servers running under low power efficiency are detected using a sliding window detection mechanism. Basically, a PM $i$ is considered power inefficient if 3 CPU utilization samples fall below 0.55 of the maximum CPU utilization. Samples are taken at intervals of 1 min and analyzed within a sliding window containing 5 CPU utilization samples. We have chosen these values based on our previous study [33], which demonstrated that these values maximize the ratio of the amount of work performed to the consumed energy.

\subsubsection{Performance deviation detection logic}

Performance deviations can occur in the two types of workloads considered. In the case of CPU-intensive applications, the contention in shared on-chip resources causes a slowdown in the execution of tasks. Several studies and experiments 
(e.g., $[8,11,22]$ ) showed that it was possible to determine the slowdown in the execution of tasks due to performance interference, with an average slowdown error of approximately $10 \%$. Eq. (7) is the slowdown $D_{i}(\tau, t)$ experienced by a CPU-intensive task $\tau$ in PM $i$.

$$
D_{i}(\tau, t)=\frac{\frac{W(\tau, t)}{\widetilde{r}_{i}(\tau)}-\frac{W(\tau, t)}{r_{i}(\tau)}}{\frac{W(\tau, t)}{r_{i}(\tau)}}+\varepsilon
$$

where $r_{i}(\tau)$ and $\widetilde{r}_{i}(\tau)$ are the expected and effectively obtained CPU resources (in Mflop/s) assigned to the task $\tau$, respectively. The parameter $r_{i}(\tau)$ is obtained through state-of-the-art slowdown meters (e.g., [8]), while $\widetilde{r}_{i}(\tau)$ is obtained by monitoring local resources and corresponds to how much CPU is being used. The performance deviation $D_{i}(\tau, t)$, that is, slowdown in the case of CPU-intensive applications, can be negative (i.e., the task is executing faster) or positive, and the same applies to the estimation error $\epsilon$ (i.e., the average error in slowdown samples plus random fluctuations).

The same logic applies to network-intensive tasks, and the performance deviation is stated by Eq. (8).

$$
D_{i}(\tau, t)=\frac{b_{i}(\tau, t)-\widetilde{b}_{i}(\tau, t)}{b_{i}(\tau, t)}
$$

where $b_{i}(\tau, t)$ and $\widetilde{b}_{i}(\tau, t)$ are the demanded and effectively assigned network I/O bandwidth (in Gbit/s) at instant $t$ to task $\tau$ running in node $i$, respectively. Due to fluctuations, the performance deviation $D_{i}(\tau, t)$ can be negative (i.e., the task is demanding for less $\mathrm{I} / \mathrm{O}$ bandwidth) or positive when the demand exceeds the assigned $\mathrm{I} / \mathrm{O}$ bandwidth.

\subsubsection{Performance enforcing logic for CPU-bound workloads}

Application QoS requirements are difficult to satisfy because of the variability of workloads and unexpected performance deviations due to interference among VMs sharing on-chip resources. To address performance deviations in CPU-intensive applications, we deploy a module combining two blocks [32]: (i) Kalman Filter (KF) noise removal and (ii) the Linear Regression-based (LR) completion estimator. The simple Kalman filter [41] is a powerful estimator that has the ability to smooth noisy data and to provide reliable estimates of signals affected by indirect, inaccurate and uncertain observations (e.g., Gaussian noise). In turn, regression analysis [35] is a common statistical procedure to model relationships between variables. Its purpose is to predict dependent variable $Y$ based on explanatory variable $X$, such that $Y=a X+b$. The module is shown in Fig. 2 .

For each task, the performance deviation estimator module leverages the KF to eliminate Gaussian distributed unpredictable disturbances in slowdown data samples provided by upstream state-of-the-art tools (e.g., [8]). At each sample time, the KF produces the estimated remaining workload using the slowdown estimation. Then, an LR is used to estimate the instant when the remaining workload is zero, i.e., the task finish time, based on the last three samples of the remaining workload along time. The intersection of the LR line with the time axis gives the estimated finish time. Henceforth, the combination of KF and LR will be referred to as the KFLR module.

\subsubsection{Performance enforcing logic for network-bound workloads}

To handle performance deviations in network-intensive workloads, the data samples containing CPU and network bandwidth resources are injected in two separate Kalman filters, as shown in Fig. 3. Then, based on the output of the Kalman filters, we define a soft limit of resources to assign for each VM. Basically, the soft limit implements a lower bound of bandwidth and CPU resource availability. The intention is to define what fraction of the bandwidth and CPU resources each VM can have when the PM is fully utilized or overloaded. Thus, in the case of resource contention among co-located VMs, the soft limit establishes the minimum resources guaranteed for each VM. In turn, a VM explores the concept of the hard limit, which implements an upper bound of resource consumption, and it is specified by the total amount of free resources in the PM. For example, if $500 \mathrm{Mbit} / \mathrm{s}$ of network bandwidth are assigned to a $V M_{1}$ (i.e., the soft limit is $500 \mathrm{Mbit} / \mathrm{s}$ ) and there is a co-hosted $V M_{2}$ that requires only $300 \mathrm{Mbit} / \mathrm{s}$ at a certain instant, $V M_{1}$ can use the remaining $200 \mathrm{Mbit} / \mathrm{s}$ from the total of $1 \mathrm{Gbit} / \mathrm{s}$ deployed by the PM, being the hard limit for PM1 $700 \mathrm{Mbit} / \mathrm{s}$. The same applies for the CPU resource. For a VM, if the Kalman filter estimates that the soft limit deviates $5 \%$ from the assigned amount of resources, the scheduling algorithm presented below is invoked to proceed with a mapping adaptation.

In this paper, KFIO henceforth represents the mechanism by which performance deviations are handled in network-intensive workloads.

\subsection{Power- and Interference-Aware Scheduling Algorithm (PIASA)}

In this section, we introduce our proposed scheduling algorithm to allocate applications in a power- and interference-aware manner. It extends the POFARE algorithm introduced in [34] by taking into account the interference among co-located tasks and the diversity of QoS requirements. The cloud manager considers power efficiency estimations and performance deviations provided by the modules described in Sections 3.3.1 and 3.3.2. Because the problem of mapping the VMs to the PMs is NP-complete, the proposed algorithm is heuristic. For the incoming applications $\left\{j_{1}, \ldots, j_{k}\right\}$, the cloud 
manager creates and manages a set of virtual clusters, one for each user, to execute the workloads associated with the applications. When invoked by the cloud manager, the algorithm selects, at each step, a VM to schedule and determines a destination PM.

\subsubsection{VM selection}

The algorithm creates a list of prioritized groups of tasks $\left\{\tau_{1}, \ldots, \tau_{l \times k}\right\}$. The priorities are, from highest to lowest: (1) performance deviation; (2) new tasks; and (3) running in a power-inefficient PM. In the first group (i.e., performance deviation), network-intensive tasks have priority over CPU-intensive tasks, and the priority is reversed for other groups. The aim is to exploit the deadline of CPU-bound workloads and provide network-intensive applications with more stability, avoiding migration overheads, so they can serve more requests successfully. Network-intensive tasks are sorted in ascending order according to the difference between resource demand and assigned resources (Eq. (8)). $D_{i}(\tau, t)$ will be negative when demand for resources is less than the assigned resources, zero when it is a new task, and positive if demand for resources is greater than the assigned resources. Thus, over-provisioned network-intensive workloads can release excessive resources to those that suffer from scarcity. In turn, CPU-intensive tasks in each group are sorted in ascending order according to their slack time (Eq. (2)). Tasks with negative slack time will be eliminated from the scheduling queue. These steps correspond to lines 3-6 of the algorithm PIASA described in Algorithm 1.

Algorithm 1. Power- and Interference-Aware Scheduling Algorithm (PIASA)

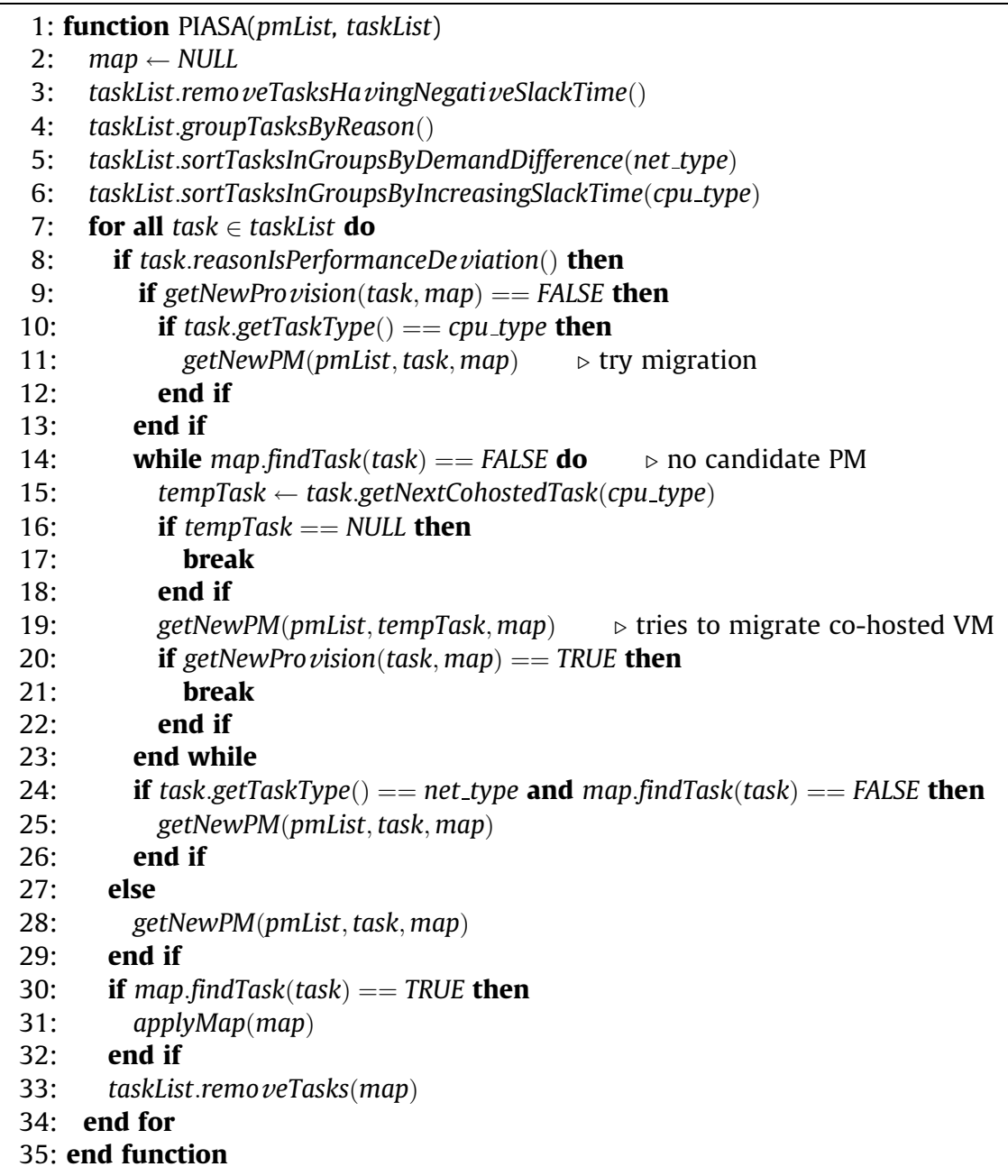

\subsubsection{VM deployment}

After a VM is selected to be scheduled from the prioritized list in line 7 of Algorithm 1, the reason for scheduling is checked at line 8, and all PMs are evaluated with respect to performance, task resource requirements, and PM power efficiency. The scheduling of tasks due to performance deviation (line 8) is achieved based on the expansion or shrinkage 


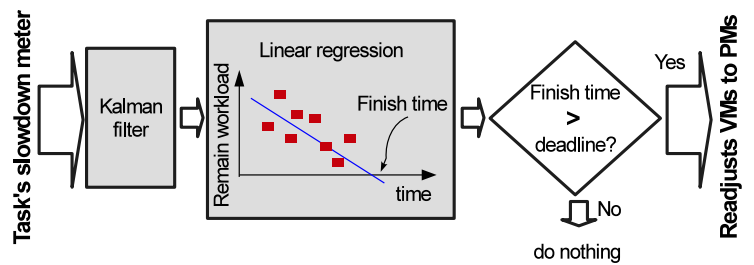

Fig. 2. Performance deviation estimator module for CPU-intensive workloads.

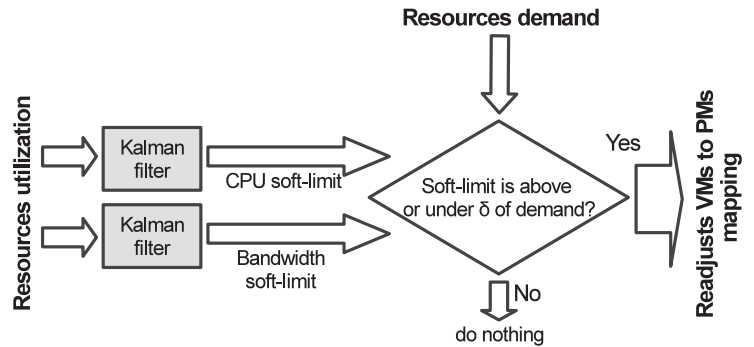

Fig. 3. Performance deviation estimator module for network-intensive workloads.

of resources (line 9). If the expansion of VMs proves to be impossible due to scarcity of resources, the algorithm proceeds in different ways according to the type of workload. For CPU-intensive workloads, the algorithm starts by trying to migrate the $\mathrm{VM}$ in line 11. If the migration overhead is greater than the slack time in line 14 (i.e., the task will not finish within the deadline), the algorithm tries to migrate co-located tasks (lines 15-19), starting with the CPU-bound task having the largest slack time until the released resources are sufficient to expand the degraded VM (line 20). For network-intensive workloads, the strategy is to migrate CPU-bound workloads (lines 15-19) less sensible to migration overheads in an attempt to free resources. If, however, freed resources are not sufficient for the network-bound application to expand in line 20, the algorithm tries to migrate the application in line 25. The function getNewPM retrieves the PM that provides the highest value for the interference-power metric (ip) given by Eq. (9):

$$
\begin{aligned}
& \text { ip }=\max \{\operatorname{Value}(i), i=1 . . h\} \\
& \text { Value }(i)= \begin{cases}E_{P_{i}} & \text { if } \sum_{\tau=1}^{n-1} r_{i}(\tau, t)<\alpha \\
I_{i} & \text { otherwise }\end{cases} \\
& \text { where } I_{i}= \begin{cases}1-\frac{\bar{D}_{i}(t)}{\max D_{h}(t)} & \text { if workload is CPU-bound and } \bar{D}_{i}(t)>0 \\
\Phi_{i}(t) & \text { otherwise }\end{cases}
\end{aligned}
$$

where $h$ is the number of servers. The Value $(i)$ is given by the power-efficiency $E_{P_{i}}$, defined in Eq. (6), if the usage resources are below $\alpha$, or by $I_{i}$, the interference factor on machine $i$. As $\alpha$ increases and approaches 1 , ip becomes more power-aware biased, which means the algorithm tends to select the most power-efficient node to schedule the task. Instead, as $\alpha$ tends to 0 , ip is interference-aware biased, and the algorithm attempts to find the node with the least interference among the qualified candidates, thus providing better conditions to fulfill the task's QoS requirements. The value of $\alpha$ can be dynamically adjusted at runtime depending on the status of the applications QoS deviations and the nodes' power consumption.

The interference factor $I_{i}$ of machine $i$ is computed by the ratio of the average slowdown $\bar{D}_{i}(t)$ experienced by $i$ over the maximum average slowdown among all machines for CPU-bound workloads with positive slowdown. Otherwise, the parameter $\Phi_{i}(t)$, expressing the factor of consolidation and interference, defined by Eq. (11), is used.

$$
\begin{aligned}
& \Phi_{i}(t)=1-\frac{(n-1)-\left|H_{i}(t)\right|+\# \text { Loads }_{\tau_{n}}}{2 \times V} \\
& \text { and } H_{i}(t)=\# \text { Loads }_{I O}-\# \text { Loads }_{C P U}
\end{aligned}
$$

where $t$ is the current time instant, $(n-1)$ is the number of VMs running in server $i, H_{i}(t)$ is the heterogeneity degree and \#Loads $\tau_{\tau_{n}}$ is the number of loads of the same type as the task $\tau_{n}$ running in the PM $i$. The \#Loads ${ }_{C P U}$ and \#Loads $s_{I O}$ are the number of CPU-bound and network $\mathrm{I} / \mathrm{O}$ loads running on machine $i$, respectively. $V$ expresses the number of VMs hosted in the PM running more VMs so that the values are normalized among all PMs evaluated. For example, if at instant $t$ a server hosts 3 CPU-bound tasks and 1 network-bound task and the cloud manager is trying to schedule a CPU-bound task, then $H_{i}(t)=-2$ and \# Loads $_{\tau_{n}}=3$. 
In this regard, $\Phi_{i}(t)$ will vary within [0,1]. As $\Phi_{i}(t)$ tends to zero, for a given machine $i$, its Value decreases, and the chance of being selected to run task $\tau_{n}$ also decreases. $\Phi_{i}(t)$ tends to zero: (a) when the number of VMs in $i$ increases, so that the system will give preference to less-loaded machines; (b) as the heterogeneity degree decreases, meaning that the machine is balanced because workloads running in the server tend to be of a different type; and (c) as the type of task $\tau_{n}$ is of the same type of tasks running on PM $i$, i.e., the system will avoid hosting tasks of predominantly the same type. This last assumption follows from the fact that the impact in the performance of co-hosted workloads is alleviated if they show dissimilar resource needs [15,19]. The denominator $2 \times V$ forces the normalization of the equation, thus ranging within [0,1].

After the machine with the highest Value is selected, designated as PM $i$, the slowdown that task $\tau_{n}$ will be subjected to depends on the load of the machine. For CPU-bound workloads, the slowdown $\beta_{i}$ is provided by Eq. (12).

$$
\beta_{i}(t)= \begin{cases}\bar{D}_{i}(t) & \text { if task } \tau_{n} \text { is new } \\ D_{i}\left(\tau_{n}, t\right) & \text { if shrinking/expanding task } \\ \max \left(D_{i}\left(\tau_{n}, t\right), \bar{D}_{i}(t)\right) & \text { if migrating task }\end{cases}
$$

where $D_{i}\left(\tau_{n}, t\right)$ is the slowdown for task $\tau_{n}$ and $\bar{D}_{i}(t)$ is the average slowdown experienced by the tasks hosted in PM $i$. Knowing $\beta_{i}(t)$, the scheduling algorithm is now able to estimate the additional amount of CPU resources $\Delta r_{i}\left(\tau_{n}, \beta_{i}(t)\right)$ to assign to task $\tau_{n}$ to mitigate the performance deviation, as expressed by Eq. (13):

$$
\Delta r_{i}\left(\tau_{n}, \beta_{i}(t)\right)=\frac{W\left(\tau_{n}, t\right) \times \beta_{i}(t)}{d_{\tau_{n}}-t-m_{e i}}
$$

where $m_{e i}=0$ for a new or a shrink/expanded task. In the case of network-intensive workloads, the additional amount of bandwidth resources $\Delta r_{i}\left(\tau_{n}, \beta_{i}(t)\right)$ to assign to task $\tau_{n}$ is given by Eq. (14) and is determined by the difference between the previously assigned resources $b_{i}\left(\tau_{n}, t\right)$ and the demand for resources estimated by the Kalman filter $\widetilde{b}_{i}\left(\tau_{n}, t\right)$ at instant $t$ :

$$
\Delta r_{i}\left(\tau_{n}, \beta_{i}(t)\right)=\widetilde{b}_{i}\left(\tau_{n}, t\right)-b_{i}\left(\tau_{n}, t\right)
$$

Next, the algorithm determines the entire amount of resources needed to fulfill the QoS requirements of tasks during runtime. In the case of CPU-intensive workloads, Eq. (15) specifies that a task $\tau$ can be deployed, or migrated, to a PM $i$ at time $t+m_{e i}$ if the following conditions are met: (i) the node provides the minimum resources $r_{i}(\tau)+\Delta r_{i}\left(\tau, \beta_{i}(t)\right)$ required by the task during the $d_{\tau}-t-m_{e i}$ time interval to execute the remaining workload $W(\tau, t)$ at instant $t$; and (ii) the required resources $r_{i}(\tau)$ are not more than $\max r(\tau)$ to be executed by its deadline.

$$
\left\{\begin{array}{l}
\left(r_{i}(\tau)+\Delta r_{i}\left(\tau, \beta_{i}(t)\right)\right) \times\left(d_{\tau}-t-m_{e i}\right) \geqslant W(\tau, t) \\
r_{i}(\tau)+\Delta r_{i}\left(\tau, \beta_{i}(t)\right) \leqslant \max r(\tau)
\end{array}\right.
$$

Finally, the scheduling algorithm checks whether candidate PM $i$ can provide the minimum resources required by task $\tau$, thus fulfilling Eq. (16) as follows:

$$
r_{i}(\tau)+\Delta r_{i}\left(\tau, \beta_{i}(t)\right)+\sum_{\tau=1}^{n-1} r_{i}(\tau) \leqslant C_{i}
$$

where $C_{i}$ represents the available capacity (i.e., CPU or network bandwidth) in node $i$. At the end, the algorithm applies a cap resource usage to assign to tasks the strictly necessary amount of CPU to complete them by the deadline and the network bandwidth to maintain the throughput. The use of resource limiting was already demonstrated to be effective in the managing of resources $[34,36]$. During execution, the cap parameter is dynamically updated to explore the available fraction of resources in the physical server.

\section{Evaluation and simulation scenario}

This section presents the metrics used to evaluate the performance of the proposed mechanism to address performance deviation and power inefficiencies. The workload characteristics and simulation scenario are described as well.

\subsection{Performance metrics}

We have defined the four main metrics to evaluate the performance of the algorithms in terms of energy efficiency and the capacity to fulfill the application's QoS requirements: (i) completion rate of users' jobs; (ii) service rate of transaction requests; (iii) ratio of useful Mflops processed to the energy consumed; and (iv) the working efficiency.

\subsubsection{Completion rate of users' jobs}

The first metric applies to CPU-intensive workloads and measures the completion rate of users' jobs. It is calculated as the ratio of the number of jobs completed by their deadline, $J_{C}$, to the number of submitted jobs, $J_{S}$. The metric is expressed as Eq. (17). 


$$
E_{J}=\frac{J_{C}}{J_{S}}
$$

where the $E_{J}$ value falls in the interval $[0,1]$ and is the SLA metric for CPU-intensive applications. The percentage of SLA violations is given by $\left(1-E_{J}\right) \times 100$.

\subsubsection{Service rate of transaction requests}

The second metric applies to network-intensive workloads and measures the percentage of SLA fulfillment by relating the number of applications that successfully served at least $95 \%$ of requests within a time unit.

$$
E_{R}=\frac{R_{C}}{R_{S}}
$$

where $R_{C}$ is the number of requests that were successfully served and $R_{S}$ is the number of submitted requests. In other words, $R_{C}$ is a measure of the average throughput of VMs at different workload rates. The $E_{R}$ value falls within the interval [0,1].

\subsubsection{Energy efficiency}

The third metric calculates the amount of energy consumed in Joules to produce useful work. By useful work, we count the number of Mflops associated with successfully completed jobs only, $J_{C}$. The energy efficiency metric $E_{M}$, shown in Eq. (19), is calculated by dividing the sum of the workloads from all tasks of successfully completed jobs by the overall energy consumption. The energy is then calculated by multiplying the average power consumption of the computing infrastructure (i.e., for all active physical nodes $u$ at all sample times $f$ ) by the number of sample times $f$, multiplied by 60 (because samples are taken per minute):

$$
\begin{aligned}
& E_{M}=\frac{\sum_{j=1}^{k} \theta_{j} \times \sum_{\tau=1}^{n} W(\tau, 0)}{\frac{\sum_{s=1}^{f} \frac{\sum_{i=1}^{u} P_{i}}{u}}{f} \times f \times 60} \\
& \theta_{j}= \begin{cases}1, & \text { if job } j \text { completed } \\
0, & \text { otherwise }\end{cases}
\end{aligned}
$$

We henceforth represent this metric as Mflops/Joule.

\subsubsection{Working efficiency}

The fourth metric determines the quantity of useful work performed (i.e., the completion rate of users' jobs) by the consumed power. This is determined by multiplying $E_{J}$ by the average power efficiency based on Eq. (6) for all active physical nodes $i \in[1, u]$ at all sample times $f$. Eq. (20) shows the calculation of the working efficiency $E_{W}$.

$$
E_{W}=\frac{\sum_{s=1}^{f} \frac{\sum_{i=1}^{u} E_{P_{i}}}{u}}{f} \times E_{J}, \quad \forall u \leqslant h
$$

Eqs. (19) and (20) express the amount of useful work performed from different perspectives. The first equation quantifies the number of useful Mflops by the consumed energy, while the second equation measures the quantity of useful work (i.e., completion rate of users' jobs) performed with the consumed power. A good algorithm must maximize both.

Maximizing the working efficiency implies diminishing the power consumption and increasing the number of completed jobs. Such a goal entails a trade-off between power-efficiency and performance interference. Power-efficiency can be achieved through a higher level of consolidation that, in turn, generally causes more performance degradation and thus negatively affects the rate of completed jobs.

\subsection{Workload and performance characteristics}

In this section, we describe the characteristics of the two types of workloads considered and of the performance interference incurred due to the consolidation of applications.

\subsubsection{Workloads based on Google cloud tracelogs}

Several recent comprehensive analyses (e.g., [28,23]) of the workload characteristics derived from Google cloud tracelogs, featuring over 900 users submitting approximately 25 million tasks over a month, yielded significant data on the characteristics of submitted workloads and the management of cluster machines. These studies enable further work on important issues in the domain of resource optimization and energy efficiency improvement. The studies show that approximately $75 \%$ of jobs only run one task, and most of the jobs have less than 28 tasks that determine the overall system throughput. The job inter-arrival time is $4 \mathrm{~s}$, with each job lasting an average of $3 \mathrm{~min}$. The jobs follow distributions such as lognormal, gamma, Weibull, or even exponential. The majority of jobs run in less than $15 \mathrm{~min}$, although there are a small number of jobs that run longer than 300 min. Moreover, the task length, number of tasks per job, and CPU utilization (varying from near 0\% to nearly 25\%) follow a lognormal distribution. Most of the tasks use less than $2.5 \%$ of the server's RAM. 
Based on these key findings, we created two sets of synthetic jobs. The first set contained 3614 CPU-intensive jobs to simulate users' non-interactive batch jobs, totaling 10,357 tasks, each with a deadline rounded up to $10 \%$ more than its minimum necessary execution time. The job deadline equals the deadline of the jobs longest task. The second set included 148 network-intensive groups of web applications and a total of 413 tasks. They are mostly network bandwidth consuming, and the required bandwidth due to oscillation in the number of requests per time unit and the file size to download varies randomly with time, following a lognormal distribution with an average value and a standard deviation of 52.5 and 265 , respectively. Ersoz et al. [9] indicate that lognormal distributions model web server workloads well. The CPU consumption changes as well but in a residual way, following a lognormal distribution with an average value and a standard deviation of 4 and 3.5, respectively. In fact, it is common to observe CPU utilization for a web workload varying in the range 2-15\% [1,2]. For both types of workloads, the runtime is specified by the user, and the VMs running them require a RAM size of 256, 512, or $1024 \mathrm{MB}$, selected randomly.

\subsubsection{Performance interference characteristics}

The behavior of co-located jobs is often described as non-deterministic and, hence, difficult to estimate [22]. However, recent research has shed some light on this subject. These studies have contributed several key findings that support the characteristics of performance interference among co-located VMs in the simulated cloud infrastructure. For example, Pu et al. [30] indicate that interference is greater when two applications of the same type are co-located, sharing the same physical resources (i.e., contending for the same resources). Interference among CPU-intensive applications occurs mainly due to contention in the LLC and memory bus [8] and can degrade performance by imposing a slowdown of as much as 120\% [11] (some authors report even larger values [13]). Lim et al. [22] observed a linear relationship between total completion time and individual completion times, a relationship that is lost when different types of jobs co-execute in the system. Verma et al. [40] observed that very large applications (in terms of working set size, i.e., the size of cache an application uses) are not affected by other workloads running on the same physical machine, and small applications do not suffer performance degradation as long as the sum of working set sizes remains less than the server's cache. Moreover, the degradation in performance of applications starts to stabilize (i.e., does not increase) when the pressure over the servers' cache is double the capacity.

Based on these key findings, we have simulated performance interference among co-located CPU-intensive workloads. Basically, each CPU-intensive application utilizes a certain amount of cache to store data during runtime. Such an amount is determined by a Poisson distribution with a mean of $13 \%$ (an acceptable value considering current processors [17]) of the total physical cache. For example, Lei et al. [21] utilized a Poisson distribution to study LLC management strategies.

The degree of performance interference of an application depends on the applications working set size and the working set size of co-hosted applications. Specifically, our simulation model implements the following key findings [11,13,24,40]: (i) interference among co-hosted applications is residual (e.g., almost zero) as long as the sum of their working set sizes is less than the cache size; (ii) the performance of an application degrades as soon it draws closer to the size of the cache, and it tends to stabilize afterward as it moves away from that size; (iii) cache contention decreases CPU throughput (instructions dispatched per second), hence imposing slowdown in the execution of applications. The performance degradation of the applications hosted in the same PM is determined by $\mathrm{e}^{-((x-1) / 2 x)^{3}}$ in the case in which the sum of the working sets size, $x$, is less than the PM cache size; and it is $\mathrm{e}^{-\left((x-1) / 2 x^{0.95}\right)}$ otherwise. These expressions were inferred from the results presented in $[24,29,40]$, and $x$ is given in percentage form, so that $x$ will be greater than $100 \%$ when the application requirements exceed the PM capacity. Because we assume that web applications are characterized mainly by network-intensive workloads, interference among co-hosted web applications is essentially caused by contention in the network I/O channel, being immune to on-chip interference.

\subsection{Comparison of strategies}

To assess the quality of the mechanism proposed in this paper, alternative mechanisms are implemented to compare their performance with that of our performance deviation estimator and scheduling algorithm.

\subsubsection{Alternative performance deviation estimators}

Three other common performance deviation detectors were implemented. For all strategies, data sample analysis is performed over a sliding window containing 5 performance deviation samples per minute, taken at constant intervals [34]. The threshold was set to $10 \%$ more than the task's minimum necessary execution time. The first, Simple ThresholD (STD) VM adaptation, is triggered if 3 slowdown data samples exceed the predefined threshold value. The second mechanism, Average ThresholD (ATD), determines the average slowdown from samples within the window, and if the average value exceeds the predefined threshold value, condition detection is triggered. The third approach, Extended ThresholD (ETD), calculates the average slowdown from samples within 3 consecutive windows (each window differing from the prior one in one sample) and invokes the scheduling algorithm if the average result exceeds the mentioned threshold.

\subsubsection{Alternative scheduling algorithms}

Garg et al. [10] proposed a dynamic resource management strategy to handle scheduling of two types of applications, namely, compute-intensive non-interactive jobs and transactional applications such as web servers. The mechanism 
responds to changes in transactional workloads and tries to maximize resource utilization while enforcing the SLA requirements and specifications. A web VM is scheduled based on best-fit, not taking into account the resources consumed by VMs running dynamic jobs. To maximize the energy efficiency, the scheduler tries to explore free resources in nodes running web applications to schedule dynamic jobs. However, if free resources are insufficient to execute the task within its deadline, the job is allocated to another node in the form of a static job. Resources are transferred from dynamic jobs to web applications as demand changes. Periodically, the algorithm verifies the SLA accomplishment and realizes consolidation. The priority of scheduling is: (1) enforce SLAs; (2) schedule the jobs from the batch job queue; and (3) consolidation.

Our work is closely related to [10] in the sense that we address the resource allocation problem within a data center that runs different types of application workloads (i.e., CPU- and network-bound applications), aiming at maximizing resource utilization and profit and ensuring that the QoS requirements of users are met as specified in SLAs. To achieve these objectives, dynamic resource allocation and consolidation are implemented. Unlike our work, Garg et al. [10] do not consider performance deviation due to contention in on-chip resources (e.g., LLC and memory bus). Moreover, our mechanism is able to (i) implement dynamic vertical elasticity of resources, (ii) make resource allocation decisions based on noisy performance deviation data samples, and (iii) balance energy efficiency and performance in the execution of applications.

\subsection{Simulation setup}

Because the target system is a cloud computing infrastructure and the functionality of re-sizing a VM dynamically, as required by the proposed resource management algorithm, is unavailable in production clouds, we resorted to simulation to evaluate the algorithms discussed in this paper. Simulation allows us to perform a statistically significant number of experiments for a wide range of application configurations in a reasonable amount of time. Due to the limitations of current simulation software programs, such as CloudSim [5], that do not support dynamic VM re-sizing, we have implemented our own discrete-event simulation software that was validated in our previous work [33,34]. Events are generated statistically following distributions observed in previous work. The workload is based on the Google Cloud trace logs (details in Section 4.2.1); the interference characteristics were derived from [24,29,40] (details in Section 4.2.2); and VM migrations follow the characteristics presented in [14].

We simulated a cloud data center infrastructure aggregating 50 homogeneous physical servers. Each server contains a CPU with a capacity assumed to be $800 \mathrm{Mflops} / \mathrm{s}$ with an electrical power consumption of $275 \mathrm{~W}$ when fully loaded. Parameters $p 1$ and $p 2$ for Eq. (5) were defined as 0.7 and 0.3 [3,4], respectively. The bandwidth of the network interfaces for a server was considered to be $1 \mathrm{Gbit} / \mathrm{s}$. The scheduling algorithm has no knowledge of when applications are submitted to the cloud or of the change in workload demands. Each VM requires a RAM size of 256, 512, or 1024 MB, randomly selected; the migration overhead of the VM depends on the memory size and the available network bandwidth in the LAN link [14], considered here as taking 8, 10 and $12 \mathrm{~s}$, respectively. In simulations considering network-intensive applications, all the tested algorithms reserved $15 \%$ for free bandwidth in every PM to better accommodate abrupt oscillations without migration.

\section{Results and analysis}

In this section, we present and discuss the results associated with the proposed mechanism to detect and mitigate performance deviations and power inefficiencies. In our simulations, we first assess the effectiveness of the proposed performance estimator and scheduling algorithm to handle CPU-bound workloads. Then, we investigate how well the mechanism performs in fulfilling network-intensive application QoS requirements and in accommodating the variations in workload demands. The last section analyses the effectiveness of the proposed mechanism in building energy-efficient virtual clusters that fulfill the dissimilar types of QoS requirements of heterogeneous applications.

\subsection{CPU-bound workloads}

This set of simulations intends to analyze the performance of the slowdown estimator and scheduling algorithm in the provision of resources to CPU-intensive jobs.

\subsubsection{Performance of the scheduling algorithms}

Table 1 shows the results for the performance of scheduling algorithms in the absence of interference among co-hosted CPU-bound workloads (i.e., the applications do not experience slowdown during runtime). Provisioning of resources considers power-efficiency optimization. Because Garg et al. [10] works based on the best-fit approach and does not consider the slowdown for scheduling of tasks, we defined the parameter $\alpha$ for Eq. (9) as 1.0 to make PIASA power-efficiency biased and to provide a fairer comparison. The results show that PIASA achieves the same completion rate of jobs as Garg et al. [10] but improves the energy efficiency and working efficiency by approximately $20.6 \%$ and $21.8 \%$, respectively. This means the PIASA strategy can produce the same work with CPU-bound workloads as Garg et al. [10] while consuming less energy, as shown in column $E(\mathrm{MJ})$ of the table. The reason is that PIASA achieves higher levels of consolidation due to the use of the cap parameter, thus using a lower average number of PMs during simulation, Avg \# PMs, and, hence, consuming less energy. 
Table 1

Performance of scheduling algorithms in the management of CPU-bound workloads, applying power optimization only (i.e., without slowdown). The $\alpha$ parameter in Eq. (9) was set to 1.0.

\begin{tabular}{llllr}
\hline Algorithm & $E_{J}(\%)$ & $E_{M}$ & $E_{W}(\%)$ & $E(\mathrm{MJ})$ \\
\hline Garg et al. [10] & 100.00 & 0.0572 & 59.23 & 114.7 \\
PIASA & 100.00 & 0.0690 & 72.15 & 7.61 \\
\hline
\end{tabular}

Table 2 registers the results for scheduling algorithms when co-hosted tasks experience performance interference. For the reason indicated above, we set up $\alpha=1.0$. The PIASA algorithm is combined with the KFLR slowdown estimator to address possible slowdown situations in the execution of tasks. The upstream slowdown meter provides slowdown data samples with an average slowdown error of $10 \%$ and a standard deviation of $20 \%$. As observed, the performance of the Garg et al. [10] algorithm degrades substantially because it ignores the slowdown in the execution of tasks due to performance interference. In turn, the PIASA strategy is able to complete more than $95 \%$ of the submitted jobs (i.e., $E_{J}$ ), largely outperforming Garg et al. [10], which is able to complete only $12.2 \%$ of jobs. Comparing these results with those for absence of performance interference (i.e., Table 1), it is obvious that PIASA now uses, on average, more physical nodes during simulation Avg \# PMs. The increase was nearly $24 \%$. Due to the slowdown in the execution of tasks, more resources are allocated to VMs to re-establish the required levels of QoS, resulting in a higher average number of active servers during simulation. If more physical servers are active, more energy is consumed. Column $E(\mathrm{MJ})$ confirms this fact, showing an increase of $34 \%$.

\subsubsection{Performance of the slowdown estimators}

This section analyses the efficacy of the proposed slowdown estimator mechanism introduced in Section 3.3.3. Simulations are carried out by combining different slowdown estimator alternatives with the PIASA algorithm, which performs better as observed in Section 5.1.1. The upstream state-of-the-art slowdown meter provides slowdown data samples with an average slowdown error of $10 \%$ and a standard deviation of $20 \%$. The $\alpha$ parameter in Eq. (9) was set to 1.0 (which represents the case in which more performance interference occurs due to a high level of consolidation to improve energy efficiency). In Fig. 4(a), we see that KFLR completes nearly $96 \%$ of jobs, which is approximately $17.9 \%$ more than LR and approximately 37.1\% more than the common STD method. The STD and ATD methods are both very sensitive to slowdown measurement fluctuations because they respond too quickly to threshold violations. As a result, nearly $38 \%$ more VM migrations are triggered compared to PIASA, which leads to an inferior rate of completed jobs due to migration overhead. In turn, ETD seems to react too late, triggering VM migrations when tasks are no longer able to be completed by their deadlines.

The KFLR achieves superior performance compared with all the other slowdown estimator techniques. Compared to LR, the better results for KFLR are from the removal of noise from slowdown data samples before their use by LR. Moreover, with KFLR, the slowdown of tasks during runtime is less than $8 \%$ on average than the slowdown evidenced by LR. Fig. 4(b) and (c) quantify the useful work performed by the consumed energy and power, respectively. Again, we observe that KFLR is the estimator that performs better, delivering more work with less energy consumption. Compared to other estimators, this property suggests that KFLR provides higher confidence levels, resulting in better decisions despite the noisy slowdown data samples and thus outputting a better jobs-completed-to-energy-consumption ratio. The STD and ATD methods present the worst results for $E_{W}$ and $E_{M}$.

\subsubsection{Performance impact of the Average Slowdown Error (ASE)}

The slowdown meter proposed by Dwyer et al. [8] has an Average Slowdown Error (ASE) of 10\%, which is the value we have used so far. Other researchers propose alternatives with different ASEs [11,22]. This section evaluates the efficacy of the proposed performance and energy efficiency enforcing mechanism (i.e., PIASA combined with KFLR) while varying ASE in data samples provided by upstream state-of-the-art slowdown meters. Table 3 shows the evolution for the $E_{J}, E_{M}, E_{W}$ performance metrics and the average estimated system slowdown $\bar{\beta}$. The results show that the higher the uncertainty in slowdown data samples provided by upstream slowdown meters, the more difficult it is to make the right decision (the produced $\bar{\beta}$ reaches higher values as uncertainty increases). This explains why the completion rate of user jobs $E_{J}$ decreases as ASEs augment, with a consequent negative impact on energy efficiency $E_{M}$ and working efficiency $E_{W}$. However, it is important to observe, based on the results, that our mechanism can be applied effectively if we consider that upstream state-of-the-art slowdown meters (e.g., [8,11,22]) can estimate the slowdown of applications with ASEs equal to or less than $10 \%$ and that SLA contract terms in real-world environments allow a mean performance degradation of 1-5\% [3].

Table 2

Performance of scheduling algorithms in the management of CPU-bound workloads, with interference among co-hosted tasks and applying power optimization (noisy slowdown samples with an average error of $10 \%$ and a standard deviation of $20 \%$ ). The $\alpha$ parameter in Eq. (9) was set to 1.0 .

\begin{tabular}{lllll}
\hline Algorithm & $E_{J}(\%)$ & $E_{M}$ & $E_{W}(\%)$ & $E(\mathrm{MJ})$ \\
\hline Garg et al. [10] & 12.2 & 0.0023 & 8.29 & 114.7 \\
PIASA & 95.60 & 0.0452 & 69.21 & 7.61 \\
\hline
\end{tabular}




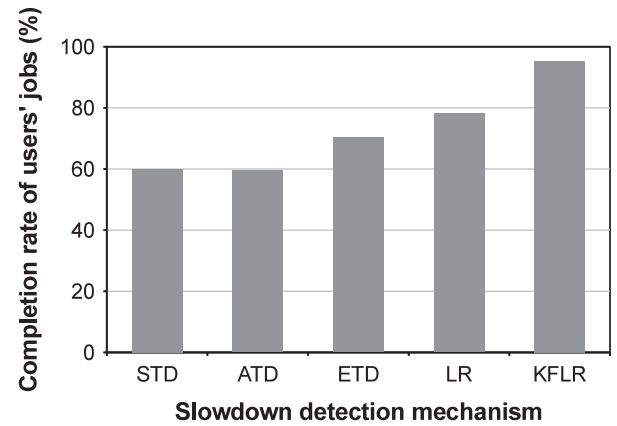

(a) Completion rate of users jobs.

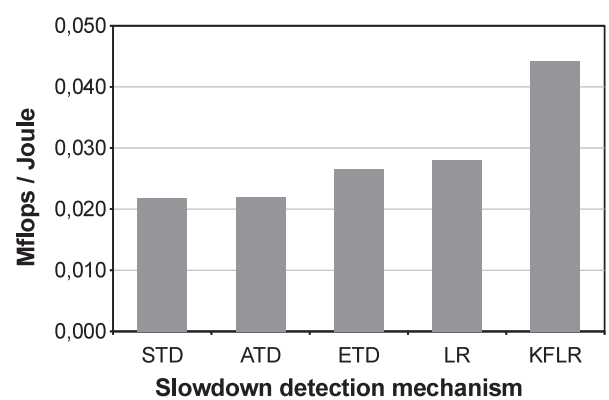

(b) Energy efficiency.

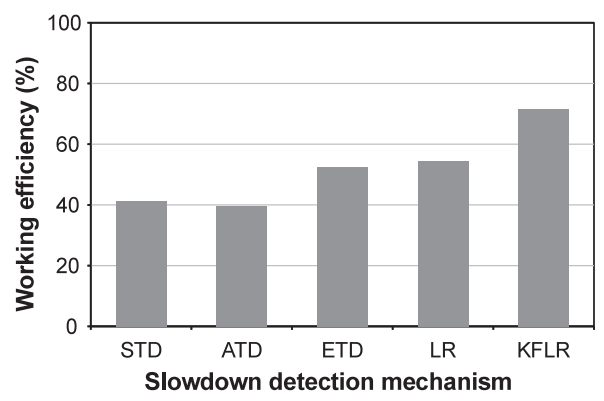

(c) Working efficiency.

Fig. 4. Comparison among slowdown estimator mechanisms in the management of CPU-bound workloads combined with the PIASA scheduling algorithm (noisy slowdown samples with an average error of $10 \%$ and a standard deviation of $20 \%$ ). The $\alpha$ parameter in Eq. (9) was set to 1.0 .

Table 3

Performance of PIASA + KFLR in the management of CPU-bound workloads while varying the average slowdown error in data samples provided by state-of-the-art upstream slowdown meters. The $\alpha$ parameter in Eq. (9) was set to 1.0 .

\begin{tabular}{cllll}
\hline ASE $(\%)$ & $E_{J}(\%)$ & $E_{M}$ & $E_{W}(\%)$ & $\bar{\beta}$ \\
\hline 5 & 97.29 & 0.0475 & 75.41 & 20.37 \\
10 & 95.60 & 0.0452 & 69.21 & 22.30 \\
15 & 92.20 & 0.0413 & 67.51 & 25.36 \\
20 & 85.50 & 0.0346 & 61.68 & 29.06 \\
25 & 73.36 & 0.0247 & 51.47 & 32.32 \\
\hline
\end{tabular}

\subsection{Network-bound workloads}

Table 4 shows the results for the performance achieved by the proposed mechanism to address network-intensive workloads. The QoS requirements are specified by users, but the characteristics of the workload demands are unknown, changing according to what is described in Section 4.2.1. The goal is to assess the efficacy of the mechanism to enforce the QoS requirements of applications for which workload demands change abruptly. Unlike Garg et al. [10], our strategy (i.e., KFIO combined with the PIASA scheduling algorithm) performs dynamic consolidation of workloads that, combined, may exceed the total available resources in the host, such as bandwidth and CPU. The cloud manager module invokes the scheduling algorithm to readjust the VM to PM mapping if the performance deviates at least $5 \%$ from the figure previously specified in the last schedule.

The results show that the PIASA algorithm outperforms the Garg et al. [10] strategy, providing sufficient resources to approximately $95.2 \%$ of applications so that they can successfully serve at least $95 \%$ of user requests. Because PIASA uses

Table 4

Performance of scheduling algorithms in the management of network-bound workloads that present oscillations in demand of resources (requested bandwidth oscillations with an average value of $0 \%$ and a standard deviation of $15 \%$ ). The $\alpha$ parameter in Eq. (9) was set to 0.50 .

\begin{tabular}{|c|c|c|c|c|c|c|}
\hline Algorithm & $100 \%$ & $\geqslant 95 \%$ & $\geqslant 90 \%$ & $\geqslant 85 \%$ & $<85 \%$ & $E(\mathrm{MJ})$ \\
\hline Garg et al. [10] & $0.0 \%$ & $3.2 \%$ & $36.1 \%$ & $30.0 \%$ & $30.7 \%$ & 262.1 \\
\hline PIASA & $37.8 \%$ & $57.4 \%$ & $2.8 \%$ & $1.5 \%$ & $0.5 \%$ & 431.5 \\
\hline
\end{tabular}


additional resources to supply the increase in user demands and thus fulfill the applications QoS requirements, achieving a higher rate of successfully served requests, more energy is consumed. This fact is reported in the last column of Table 4.

In Fig. 5, the bandwidth characteristics during a fraction of the runtime of a VM hosting a web application are plotted. The $y$-axis represents the percentage of bandwidth of the physical server the VM utilizes, and the simulation time, for which each time unit corresponds to 11 real seconds, is plotted on the $x$-axis. The bandwidth varies with time, according to the number of requests per time unit and the size of the file to download, as described in Section 4.2.1. The "Demanded" bandwidth accounts for the bandwidth required at each time unit. The "Utilized" bandwidth represents the bandwidth the application was able to use during runtime (a VM is able to use more than the initially assigned amount, as long as the physical server contains free resources), while the soft limit expresses the minimum guaranteed bandwidth the algorithm decided to assign to the application. "Scarcity" is the difference between the resources demanded and utilized, and "Wastage" is the difference between the soft limit and the demanded resources.

Fig. 5 also shows that the proposed mechanism is able to detect performance deviations that can be caused by: (1) scarcity of resources, when applications demand more resources than are initially assigned, or (2) wastage of resources, if initially assigned resources exceed application needs. For example, the line "Scarcity" was always kept at 0, which indicates that the mechanism was able to expand the resources assigned to the VM. Even in the case of the abrupt increase in "Demand" that occurred between interval 1060 and 1070, the proposed mechanism proved to be effective in reacting on time by assigning more resources, as demonstrated by the "Soft limit" line. In addition, the mechanism demonstrated awareness of the excessive amount of resources assigned to the VM, as indicated by the "Soft limit" line, which falls during application runtime. For this case, the "Wastage" line demonstrated that $95.6 \%$ of the time, the excess of resources was less than or equal to $5 \%$, and wasted resources of only $2 \%$ or lower were observed $74.6 \%$ of the time.

Fig. 6 draws the CPU resource characteristics for the same fraction of the runtime of the VM hosting the web application. The $y$-axis represents the percentage of CPU of the physical server the VM utilizes, and the $x$-axis contains the simulation time. The CPU resource consumption varies with time according to workload needs. The figure shows that the proposed strategy successfully fulfilled the workload demand in terms of CPU. Moreover, in the specific case of this network-intensive application, the mechanism did not miss or waste any CPU capacity, which in the last case means that freed resources were available for other applications.

\subsection{Mixture of CPU- and network-bound workloads}

This section assesses the efficacy of the proposed mechanism to enforce the diverse QoS requirements of applications with heterogeneous workloads while optimizing the energy efficiency of the system. The process of provisioning of resources considers two types of workloads: CPU- and network-intensive workloads. The application QoS requirements are specified by users, but the change in workload demands and performance deviations during runtime are unknown and follow the characteristics described in Section 4.2.1. Because the level of consolidation is very low for $\alpha=0$, the effects produced by the mechanism to optimize the power-efficiency are irrelevant. In this sense, the consolidation mechanism was switched off for $\alpha=0$, thus stabilizing the whole system by restricting the number of migrations for power-efficiency reasons.

Table 5 shows the impact of the variation in parameter $\alpha$ of Eq. (9) in the performance of the proposed mechanism (KFLR + KFIO + PIASA). The $\bar{H}$ stands for the average load heterogeneity degree (a concept that was initially described in Section 3.4.2), measured for all servers along the simulation. As mentioned previously, as $\alpha$ tends to 1 , the mechanism tends to be more power aware and hence concerned with power-efficiency. In turn, as $\alpha$ tends to 0 , the mechanism is interference-aware biased, hence prioritizing the fulfillment of application QoS requirements over power-efficiency objectives. The results demonstrate the trade-off between the consolidation of VMs to improve power-efficiency versus higher

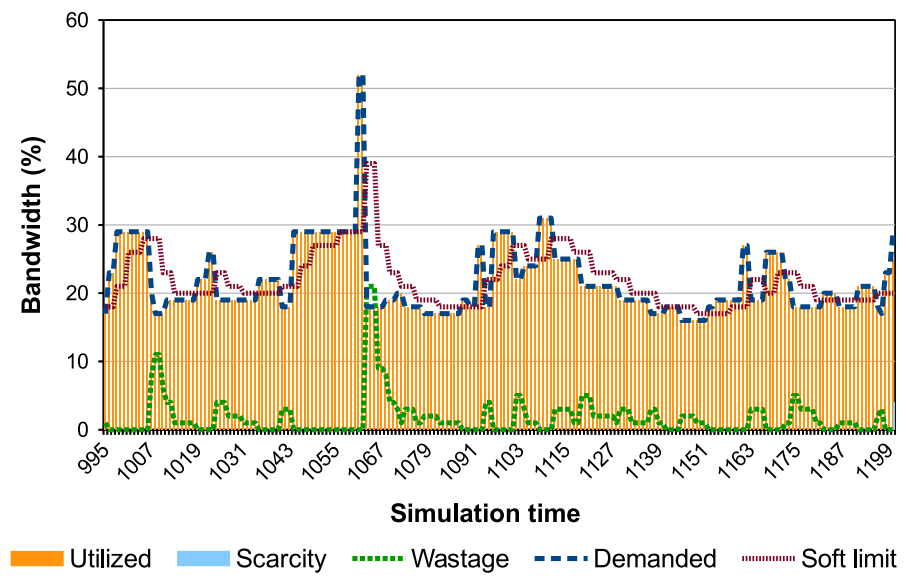

Fig. 5. Dynamic adjustment of bandwidth for a VM running a network-bound workload. The deviation threshold beyond which an adjustment was triggered was set to $\delta=5 \%$. The $\alpha$ parameter in Eq. (9) was set to 0.50 . 


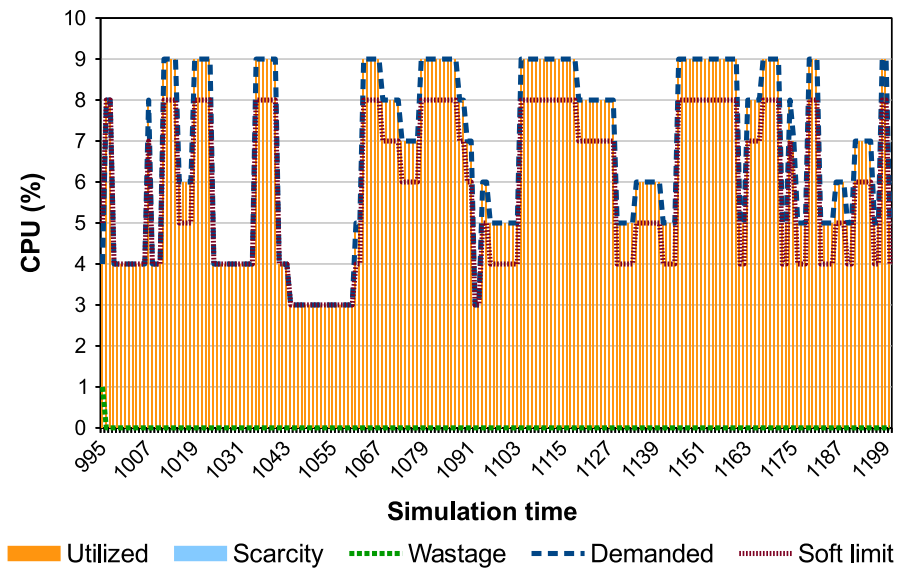

Fig. 6. Dynamic adjustment of CPU for a VM running a network-bound workload. The deviation threshold beyond which an adjustment was triggered was set to $\delta=5 \%$. The $\alpha$ parameter in Eq. (9) was set to 0.50 .

Table 5

Performance of proposed KFLR (CPU-intensive workloads) + KFIO (network-intensive workloads) + PIASA in the managing of applications. The deviation threshold beyond which an adjustment was triggered for network-intensive workloads was set to $5 \%$. The $\alpha$ parameter in Eq. ( 9 ) is varied from 1.00 to 0.00 . Because the level of consolidation is very low for $\alpha=0.0$, the consolidation mechanism was switched off in this case.

\begin{tabular}{llrlllll}
\hline$\alpha$ & $E_{R}(\%)$ & $E_{J}(\%)$ & $V M s / P M$ & $M i g(\%)$ & $\bar{H}$ & $E(\mathrm{MJ})$ & $E_{M}(\mathrm{Mflops} / \mathrm{J})$ \\
\hline 1.00 & 86.9 & 95.3 & 9.14 & 28.7 & -0.95 & 396.7 & 0.020 \\
0.75 & 93.5 & 96.3 & 8.23 & 17.9 & -0.93 & 433.2 \\
0.50 & 95.6 & 98.0 & 7.26 & 12.2 & -0.87 & 480.5 \\
0.25 & 95.6 & 99.2 & 6.66 & 8.5 & -0.80 & 512.5 \\
0.00 & 96.4 & 100.0 & 4.64 & 1.0 & -0.04 & 608.1 & 34.53 \\
\hline
\end{tabular}

levels of fulfillment of application QoS needs. For example, in the case of $\alpha=1.00$, which leads Eq. (9) to be power-efficiency biased, the mechanism achieves the best results in terms of the relationship between Mflops per Joule $E_{M}$ and work produced (i.e., completion rate of jobs) for average power consumption $E_{W}$. However, the amount of CPU-intensive jobs completed $E_{J}$ and the number of network-intensive applications successfully serving more than $95 \%$ of requests $E_{R}$ are the lowest for all $\alpha$, representing $95.3 \%$ and $86.9 \%$, respectively. Two factors explain these results for $E_{J}$ and $E_{R}$. The first regards the consolidation level VMs/PM, which increases as $\alpha$ evolves from 0 to 1 . Performance interference follows the same tendency, as more tasks sharing the node's resources represent more interference. The second factor relates to the higher average heterogeneity level value $\bar{H}$ observed during application runtime, which means that more workloads of the same type are consolidated per node, thus increasing performance interference. The variable $\bar{H}$ becomes negative in the three sets of experiments because, most of the time, the PMs ran more CPU-intensive workloads than network-intensive workloads. In turn, when the parameter $\alpha$ is 0 , the mechanism indeed proves to be interference-aware biased, leading to a decrease in the $\bar{H}$, which means there is extra care in hosting CPU- together with network-intensive workloads. Furthermore, the number of VMs successfully serving more than $95 \%$ of the network requests $E_{R}$ increases to $96.4 \%$. The completion rate of user jobs $E_{J}$ increases to $100 \%$. The consolidation of VMs (i.e., VMs/PM) diminishes, which leads in general to a minor degree of performance interference among co-hosted workloads (less contention in resources) while producing an increase in energy consumption. Accordingly, the number of migrations Mig decreases, and thus, there is greater stability in the whole system. The relationship between work produced and average power $E_{W}$ and energy $E_{M}$ consumption decreases as well.

Based on these results, we can conclude that high values of $\alpha$ are cloud provider friendly in the sense that they increase the relationship of work produced and energy consumption at the expense of a lower rate of completed network IO-intensive applications and fewer VMs serving more than 95\% of CPU-intensive requests. In turn, low values of $\alpha$ are cloud consumer friendly because they ensure higher rates of SLA accomplishment by increasing the rate of completed CPU-intensive applications and the number of VMs serving more than 95\% of network requests, at the expense of higher rates of energy consumption. In any case, if $\alpha$ is defined as 0.50 , it will represent a savings of nearly $21 \%$ in energy consumption compared to the case of $\alpha=0$, while the QoS requirements for both types of workloads will be above $95 \%$.

\section{Conclusions}

Cloud data centers apply virtualization because of the benefits it offers, which includes fault isolation and improved manageability through dynamic resource provisioning and live migration of VMs. However, virtualization technology does 
not provide performance isolation. Consequently, the phenomenon of performance interference occurs among co-hosted VMs that share physical resources. In the specific case of CPU-intensive workloads, contention in on-chip resources (e.g., LLC and memory bus) initiates performance deviation in the form of slowdown in the execution of applications. This problem is even more severe when cloud providers consolidate VMs to reduce energy consumption and operational costs and when the application workload characteristics are diverse and have different QoS requirements.

In this paper, we proposed a performance and energy efficiency enforcing mechanism composed of a performance deviation estimator and a scheduling algorithm. The objective is to guarantee diverse application QoS requirements while maximizing the energy efficiency during runtime. The tests were conducted by injecting two types of applications, CPU- and network-intensive applications. The workload characteristics of both sets follow the latest version of the Google cloud tracelogs. In the case of network-intensive applications, the number of requests per time unit and file size to download followed a lognormal distribution. In the first set of simulations, we analyzed the efficacy of the performance and energy efficiency enforcing mechanism in scheduling CPU-intensive workloads, with and without performance interference among VMs. The results show that the proposed PIASA algorithm outperformed the alternative algorithm, completing $95.6 \%$ of the submitted jobs in the presence of performance interference. In the absence of performance deviation, PIASA proved able to produce at least the same amount of work as the alternative state-of-the-art algorithm while consuming less energy. In the second set of simulations, the mechanism proved capable of ensuring that nearly $96 \%$ of network-intensive applications could successfully serve more than $95 \%$ of requests. Finally, the proposed mechanism was tested with a mixture of CPUand network-bound workloads. Considering that in real-world environments the SLA contract terms allows a mean performance degradation of $1-5 \%$, our proposed performance enforcing mechanism can be applied effectively to fulfill the expected QoS of applications and reduce energy costs by as much as $21 \%$.

Future work will consider the adaptation of a cloud middleware, such as OpenStack or OpenNebula, to support dynamic VM re-sizing in order to experimentally validate the algorithm proposed in this paper. We plan to consider other types of workloads with different resource demands and other sources of performance interference and to include additional control actuators so that we can address memory-bound applications as well. Cloud computing assists the deployment of modern applications with variable workloads, which implies that VM placement should be optimized continuously in an online manner. Sharing and contention of other resources, such as disks and scheduler parameters, also result in performance interference. Another relevant problem is the consideration of heterogeneous resources and processing workflows, in which tasks follow a sequence with mandatory precedence.

\section{Acknowledgment}

This work is partially supported by EU under the COST Program Action IC1305: Network for Sustainable Ultrascale Computing (NESUS) and by Austrian Science Fund (FWF) project TRP 237-N23: "Workflows on Manycore Processors".

\section{References}

[1] T.F. Abdelzaher, K.G. Shin, N. Bhatti, Performance guarantees for web server end-systems: a control-theoretical approach, IEEE Trans. Parallel Distrib. Syst. 13 (1) (2002) 80-96.

[2] P. Barford, M. Crovella, Generating representative web workloads for network and server performance evaluation, ACM SIGMETRICS Perform. Eval. Rev. 26 (1) (1998).

[3] A. Beloglazov, J. Abawajy, R. Buyya, Energy-aware resource allocation heuristics for efficient management of data centers for cloud computing, Future Gen. Comput. Syst. 28 (5) (2012) 755-768.

[4] A. Berl, E. Gelenbe, M. Di Girolamo, G. Giuliani, H. De Meer, M.Q. Dang, K. Pentikousis, Energy-efficient cloud computing, Comput. J. 53 (7) (2009) 10451051.

[5] R.N. Calheiros, R. Ranjan, A. Beloglazov, C.A.F.D. Rose, R. Buyya, CloudSim: a toolkit for modeling and simulation of cloud computing environments and evaluation of resource provisioning algorithms, Softw.: Pract. Exper. 41 (1) (2011) 23-50.

[6] D. Carrera, M. Steinder, I. Whalley, J. Torres, Enabling resource sharing between transactional and batch workloads using dynamic application placement, in: 9th ACM/IFIP/USENIX International Conference on Middleware, December 2008, pp. 203-222.

[7] R.C. Chiang, H.H. Huang, TRACON: interference-aware scheduling for data-intensive applications in virtualized environments, in: International Conference for High Performance Computing, Networking, Storage and Analysis, 2011, pp. 1-12.

[8] T. Dwyer, A. Fedorova, S. Blagodurov, M. Roth, F. Gaud, J. Pei, A practical method for estimating performance degradation on multicore processors, and its application to HPC workloads, in: International Conference in High Performance Computing, Networking, Storage and Analysis, November 2012, pp. $1-11$.

[9] D. Ersoz, M.S. Yousif, C.R. Das, Characterizing network traffic in a cluster-based, multi-tier data center, in: 27th International Conference on Distributed Computing Systems, June 2007, pp. 59-59.

[10] S.K. Garg, A.N. Toosi, S.K. Gopalaiyengar, R. Buyya, SLA-based virtual machine management for heterogeneous workloads in a cloud datacenter, J. Network Comput. Appl. 45 (2014) 108-120.

[11] S. Govindan, J. Liu, A. Sivasubramaniam, A. Kansal, Cuanta: quantifying effects of shared on-chip resource interference for consolidated virtual machines, in: 2nd ACM Symposium on Cloud Computing, 2011, pp. 1-14.

[12] A. Greenberg, J. Hamilton, D.A. Maltz, P. Patel, The cost of a cloud: research problems in data center networks, ACM SIGCOMM Comput. Commun. Rev. 39 (1) (2009) 68-73.

[13] Y. Hashimoto, K. Aida, Evaluation of performance degradation in HPC applications with VM consolidation, in: 3rd International Conference on Networking and Computing (ICNC), December 2012, pp. 273-277.

[14] M.R. Hines, K. Gopalan, Post-copy based live virtual machine migration using adaptive pre-paging and dynamic self-ballooning, in: ACM International Conference on Virtual Execution Environments, 2009, pp. 51-60.

[15] Q. Huang, P.P.C. Lee, An experimental study of cascading performance interference in a virtualized environment, ACM SIGMETRICS Perform. Eval. Rev. 40 (4) (2013) 43-52. 
[16] A. Iosup, S. Ostermann, N. Yigitbasi, R. Prodan, T. Fahringer, D. Epema, Performance analysis of cloud computing services for many-tasks scientific computing, IEEE Trans. Parallel Distrib. Syst. 22 (6) (2010) 931-945.

[17] A. Jaleel, M. Mattina, B. Jacob, Last level cache (LLC) performance of data mining workloads on a CMP-a case study of parallel bioinformatics workloads, in: 12th IEEE International Symposium on High-Performance Computer Architecture, February 2006, pp. 88-98.

[18] M. Keller, D. Meister, A. Brinkmann, C. Terboven, C. Bischof, eScience cloud infrastructure, in: Proceedings of the 37th EUROMICRO Conference on Software Engineering and Advanced Applications, Finland, 2011, pp. 188-195.

[19] Y. Koh, R. Knauerhase, P. Brett, M. Bowman, Z. Wen, C. Pu, An analysis of performance interference effects in virtual environments, in: International Symposium on Performance Analysis of Systems and Software, April 2007, pp. 200-209.

[20] G. Kousiouris, T. Cucinotta, T. Varvarigou, The effects of scheduling, workload type and consolidation scenarios on virtual machine performance and their prediction through optimized artificial neural networks, J. Syst. Softw. 84 (8) (2011) 1270-1291.

[21] L. Lei, A. Huiyao, Z. Peng, Study on last-level cache management strategy of the chip multi-processor, Appl. Math. Inform. Sci. 9 (2L) (2015) 661-670.

[22] S.H. Lim, J.S. Huh, Y. Kim, G.M. Shipman, C.R. Das, D-factor: a quantitative model of application slow-down in multi-resource shared systems, ACM SIGMETRICS Perform. Eval. Rev. 40 (1) (2012) 271-282.

[23] Z. Liu, S. Cho, Characterizing machines and workloads on a Google cluster, in: International Conference on Parallel Processing Workshops, Pittsburgh, 2012, pp. 397-403.

[24] J. Mars, L. Tang, K. Skadron, M. Lou Soffa, Increasing utilization in modern warehouse-scale computers using bubble-up, Micro, IEEE 32 (3) (2012) 88-99.

[25] M. Maurer, I. Brandic, R. Sakellariou, Adaptive resource configuration for Cloud infrastructure management, Future Gen. Comput. Syst. 29 (2) (2013) $472-487$.

[26] Y. Mei, L. Liu, X. Pu, S. Sivathanu, X. Dong, Performance analysis of network I/O workloads in virtualized data centers, IEEE Trans. Serv. Comput. 6 (1) (2011) 48-63.

[27] D. Meisner, B.T. Gold, T.F. Wenisch, PowerNap: eliminating server idle power, in: 14th International Conference on Architectural Support for Programming Languages and Operating Systems, Washington, 2009, pp. 205-216.

[28] I.S. Moreno, P. Garraghan, P. Townend, J. Xu, Analysis, modeling and simulation of workload patterns in a large-scale utility cloud, IEEE Trans. Cloud Comput. 2 (2) (2014) 208-221.

[29] R. Nathuji, A. Kansal, A. Ghaffarkhah, Q-Clouds: managing performance interference effects for QoS-aware clouds, in: European Conference on Computer Systems, April 2010, pp. 237-250.

[30] X. Pu, L. Liu, Y. Mei, S. Sivathanu, Y. Koh, C. Pu, Understanding performance interference of I/O workload in virtualized cloud environments, in: IEEE 3rd International Conference on Cloud Computing (CLOUD), 2010, pp. 51-58.

[31] J. Rao, Y. Wei, J. Gong, C.Z. Xu, QoS guarantees and service differentiation for dynamic cloud applications, IEEE Trans. Network Serv. Manage. 10 (1) (2013) 43-55.

[32] A.M. Sampaio, J.G. Barbosa, Estimating effective slowdown of tasks in energy-aware clouds, in: 12th IEEE Parallel and Distributed Processing with Applications (ISPA), 2014, pp. 101-108.

[33] A.M. Sampaio, J.G. Barbosa, Optimizing energy-efficiency in high-available scientific cloud environments, in: IEEE International Conference on Cloud and Green Computing, Karlsruhe, 2013.

[34] A.M. Sampaio, J.G. Barbosa, Towards high-available and energy-efficient virtual computing environments in the cloud, Future Gen. Comput. Syst. 40 (2014) 30-43.

[35] G.A. Seber, A.J. Lee, Linear Regression Analysis, vol. 936, John Wiley \& Sons, 2012.

[36] M. Silva, K.D. Ryu, D. Da Silva, VM performance isolation to support QoS in cloud, in: 26th Parallel and Distributed Processing Symposium Workshops \& PhD Forum (IPDPSW), May 2012, pp. 1144-1151.

[37] M. Stillwell, F. Vivien, H. Casanova, Dynamic fractional resource scheduling versus batch scheduling, IEEE Trans. Parallel Distrib. Syst. 23 (3) (2012) $521-529$.

[38] C. Vecchiola, S. Pandey, R. Buyya, High-performance cloud computing: a view of scientific applications, in: 10th International Symposium on Pervasive Systems, Algorithms, and Networks, Taiwan, December 2009, pp. 4-16.

[39] A. Verma, G. Dasgupta, T.K. Nayak, P. De, R. Kothari, Server workload analysis for power minimization using consolidation, in: 2009 Conference on USENIX Annual Technical Conference, 2009, pp. 1-14.

[40] A. Verma, P. Ahuja, A. Neogi, Power-aware dynamic placement of HPC applications, in: 22nd Annual International Conference on Supercomputing, June 2008, pp. $175-184$.

[41] G. Welch, G. Bishop, An Introduction to the Kalman Filter, University of North Carolina, North Carolina, 1995.

[42] J. Xu, J. Fortes, A multi-objective approach to virtual machine management in datacenters, in: 8th ACM International Conference on Autonomic Computing, Karlsruhe, 2011, pp. 225-234.

[43] X. Zhang, J.J. Lu, X. Qin, X.N. Zhao, A high-level energy consumption model for heterogeneous data centers, Simul. Model. Pract. Theory 39 (December) (2013) 41-55. 